\title{
Factors associated with burnout among US neurosurgery residents: a nationwide survey
}

\author{
Frank J. Attenello, MD, ${ }^{1}$ lan A. Buchanan, MD, ${ }^{1}$ Timothy Wen, MD, MPH, ${ }^{1}$ Daniel A. Donoho, MD, ${ }^{1}$ \\ Shirley McCartney, PhD, ${ }^{2}$ Steven Y. Cen, PhD, ${ }^{1}$ Alexander A. Khalessi, MD, ${ }^{3}$ \\ Aaron A. Cohen-Gadol, MD, MSc, ${ }^{4}$ Joseph S. Cheng, MD, MS, ${ }^{5}$ William J. Mack, MD, ${ }^{1}$ \\ Clemens M. Schirmer, MD, ${ }^{6}$ Karin R. Swartz, MD, ${ }^{7}$ J. Adair Prall, MD, ${ }^{8}$ Ann R. Stroink, MD, ${ }^{9}$ \\ Steven L. Giannotta, MD, ${ }^{1}$ and Paul Klimo Jr., MD, MPH ${ }^{10}$
}

1'Department of Neurosurgery, University of Southern California Keck School of Medicine, Los Angeles, California; ${ }^{2}$ Department of Neurosurgery, Oregon Health \& Science University, Portland, Oregon; ${ }^{3}$ Department of Neurosurgery, University of California, San Diego, California; ${ }^{4}$ Goodman Campbell Brain and Spine, Department of Neurosurgery, Indiana University, Indianapolis, Indiana; ${ }^{5}$ Department of Neurosurgery, University of Cincinnati College of Medicine, Cincinnati, Ohio; ${ }^{6}$ Geisinger Health System, Wilkes-Barre, Pennsylvania; ${ }^{7}$ Department of Neurosurgery, Medical College of Wisconsin, Milwaukee, Wisconsin; ${ }^{8}$ Department of Neurosurgery, Littleton Adventist Hospital, Littleton, Colorado; ${ }^{9}$ Central Illinois Neuro Health Science, Bloomington, Illinois; and ${ }^{10}$ Department of Neurosurgery, University of Tennessee Health Science Center, Memphis, Tennessee

\begin{abstract}
OBJECTIVE Excessive dissatisfaction and stress among physicians can precipitate burnout, which results in diminished productivity, quality of care, and patient satisfaction and treatment adherence. Given the multiplicity of its harms and detriments to workforce retention and in light of the growing physician shortage, burnout has garnered much attention in recent years. Using a national survey, the authors formally evaluated burnout among neurosurgery trainees.
\end{abstract}

METHODS An 86-item questionnaire was disseminated to residents in the American Association of Neurological Surgeons database between June and November 2015. Questions evaluated personal and workplace stressors, mentorship, career satisfaction, and burnout. Burnout was assessed using the previously validated Maslach Burnout Inventory. Factors associated with burnout were determined using univariate and multivariate logistic regression.

RESULTS The response rate with completed surveys was $21 \%(346 / 1643)$. The majority of residents were male $(78 \%)$, 26-35 years old (92\%), in a stable relationship (70\%), and without children (73\%). Respondents were equally distributed across all residency years. Eighty-one percent of residents were satisfied with their career choice, although $41 \%$ had at some point given serious thought to quitting. The overall burnout rate was $67 \%$. In the multivariate analysis, notable factors associated with burnout included inadequate operating room exposure (OR 7.57, $p=0.011$ ), hostile faculty (OR 4.07, $p=0.008)$, and social stressors outside of work (OR 4.52, $p=0.008)$. Meaningful mentorship was protective against burnout in the multivariate regression models (OR 0.338, $p=0.031$ ).

CONCLUSIONS Rates of burnout and career satisfaction are paradoxically high among neurosurgery trainees. While several factors were predictive of burnout, including inadequate operative exposure and social stressors, meaningful mentorship proved to be protective against burnout. The documented negative effects of burnout on patient care and health care economics necessitate further studies for potential solutions to curb its rise.

https://thejns.org/doi/abs/10.3171/2017.9.JNS17996

KEY WORDS national survey; physician burnout; career satisfaction; neurosurgery; residency training

$\mathrm{T}$ HE practice of medicine has undergone a radical transformation whereby the doctor-patient relationship has been displaced by remuneration, patient satisfaction, and outcome-based metrics. Coincident with this paradigm shift is a dramatic rise in bureaucratic over- sight, accountability, clinician workload, and reported rates of physician dissatisfaction. There is ever-growing concern for physician well-being in light of mounting evidence that more than half of American physicians, regardless of their career stage, exhibit signs of burnout. ${ }^{20,56,58}$ Burnout dimin-

ABBREVIATIONS AANS = American Association of Neurological Surgeons; CSNS = Council of State Neurosurgical Societies; MBI = Maslach Burnout Inventory . SUBMITTED April 20, 2017. ACCEPTED September 25, 2017.

INCLUDE WHEN CITING Published online February 9, 2018; DOI: 10.3171/2017.9.JNS17996. 
ishes access to medical care as affected physicians show more absenteeism, are more likely to curtail hours, or, worse yet, shutter their practices altogether for retirement at a time when the US physician shortage is already in dire straits..$^{21,68}$ Furthermore, burnout and other measures of overall psychological health are directly linked not only to productivity, but also to quality of care and patient satisfaction and adherence to treatment regimens. ${ }^{15,17,28,39,59,60,69}$

Burnout is the by-product of unchecked professional and emotional distress in the context of workplace dissatisfaction. ${ }^{37}$ In 1974 psychologist Herbert Freudenberger first described "staff burnout" as a state of vital exhaustion in the workplace that culminates in readily recognizable behavioral traits..$^{30}$ Christina Maslach later defined burnout as a syndrome characterized by the triad of emotional exhaustion, depersonalization, and a low sense of personal accomplishment. ${ }^{40}$ It generally develops in individuals whose occupation brings them into perpetual human contact, with emotional exhaustion appearing first. Here, the subject lacks the mental fortitude to impart emotional support to others. Gradually, there is psychological isolation until cynicism and detachment in interpersonal relations emerge as a coping strategy. In its earliest stages, burnout can coexist with many necessary clinical merits, such as empathy and compassion. However, if left unattended, burnout erodes the foundation of these attributes and eventually supersedes them. Burnout can be accompanied by physical or psychological manifestations (for example, insomnia, appetite changes, headaches, and irritability, among others) in much the same way as depression. ${ }^{34}$ However, unlike the latter's global impact on a person, burnout disrupts how an individual interfaces with their work environment. Thus, the two are distinct clinical entities, even though burnout can degenerate into depression. ${ }^{25}$

Nevertheless, burnout is not a normal eventuality in the course of daily occupational stress and personal sacrifice. Rather, it is an adverse consequence of discordance that ensues when a clinician's emotional distress is not sufficiently mitigated by the intrinsic reward system of practicing medicine or hobbies and physical exercise. ${ }^{23}$ Ultimately, there is a loss of meaning and purpose in the role as health care provider. Factors that have often been implicated in burnout include excessive work hours or call requirements, loss of autonomy, and large amounts of workhome interference. ${ }^{9,18}$ Nowhere is the stage more aptly set for burnout than in residency training, where these factors all hold true. Although there is considerable variation across specialties, residency burnout rates are reportedly twice as high for physicians in training than for their postgraduate counterparts. ${ }^{22,57}$ In one series, $76 \%$ of surveyed residents exhibited signs of burnout. ${ }^{34,57}$ Even more alarming, studies have suggested that burnout appears as early as in medical school. ${ }^{13,19}$ These findings underscore the pervasiveness of burnout among health care professionals and would seem to suggest an epidemic is underway.

Few studies have addressed burnout in a field as technically and mentally onerous as neurosurgery. In a recent national survey of practicing neurosurgeons, $57 \%$ of respondents had documented signs of burnout, although $70 \%$ would choose the same career if given the choice, attesting to the immensely rewarding nature of the spe- cialty. ${ }^{41}$ Deeply concerning, however, is the fact that only $36 \%$ would ever recommend neurosurgery as a career to their offspring. This datum echoes a widespread sentiment across the profession of medicine, in which the majority would not recommend their career choice to others. ${ }^{42}$ Such deterrence poses a considerable challenge to workforce recruitment and, ultimately, the viability and sustainability of health care. Studies on career satisfaction and burnout are therefore instrumental in identifying factors that precipitate psychological distress so that reforms can be instituted to stem the tide of disgruntled American physicians.

Here we present the results of a nationwide survey analyzing the extent of resident burnout, career satisfaction, and other quality of life variables related to physician well-being.

\section{Methods}

A modified version of the attending neurosurgeon burnout survey used by McAbee et al. ${ }^{41}$ was provided electronically via the SurveyMonkey platform. While questions paralleled the format of the prior attending-based survey, the questions significantly differed to target the resident population (Appendix Table). We conducted the survey through a SurveyMonkey questionnaire linked to the American Association of Neurological Surgeons (AANS) listserv to ensure that each resident received a unique and confidential online survey link. Each individual link was de-identified with a unique 10-digit identification assigned with no capability of tracing results to a respondent. Importantly, once a survey was submitted from an individual link, the questionnaire link became inactive, ensuring that no individual response duplications occurred.

The survey consisted of 86 questions, including 4 freetext questions and 22 questions from the previously validated Maslach Burnout Inventory (MBI). ${ }^{40}$ An electronic invitation was sent to all neurosurgical resident trainee members of the AANS on behalf of the Council of State Neurosurgical Societies (CSNS). The survey invitation was sent on 3 separate occasions between June and November 2015. Electronic communication with survey respondents consisted of a cover letter specifying study objectives and an individualized and de-identified link to the questionnaire. The introductory email invitation specified that each response would be coded in a de-identified fashion with no individual identifiers and strict confidentiality enforced. Critical email wording was as follows:

\section{Dear Resident:}

The Council of State Neurosurgical Societies (CSNS), the socioeconomic arm of the American Association of Neurological Surgeons (AANS) and Congress of Neurological Surgeons (CNS), is conducting a nationwide survey of current neurosurgical residents identifying predictors of job satisfaction, stress and burnout.

This online survey should take less than five minutes to complete. This survey is strictly confidential with every response made anonymous.

Neurosurgery is arguably one of the more mentally and physically demanding fields in medicine. A similar study conducted among practicing neurosurgeons was recently pub- 
lished in the Journal of Neurosurgery. Though there are many studies on burnout and job satisfaction in almost all fields of medicine, few studies target residents. We hope to identify predictors of satisfaction/dissatisfaction not only among resident neurosurgeons as a whole, but within various subgroups, such as neurosurgeons of different age groups, geographic locations and training levels. Identities will remain anonymous and may be pooled in a de-identified cohort for survey again in two to three years to monitor changes in opinions over time.

Participation was encouraged by program coordinators but not mandatory. This national survey was promoted and funded by the CSNS.

\section{Questionnaire Composition}

The survey included questions on key demographics, including age, sex, relationship status, number of children, and postgraduate year of training. General questions were aimed to determine whether respondents would choose their specialty or residency program if presented with the choice again, goals after residency, and prior considerations of quitting training or leaving medicine entirely. Historical questions ascertained whether respondents had completed a subinternship in their program, felt they had an adequate perception of the field prior to applying, or had spent time away from education on research or other ventures before embarking on training. Program-related questions included the position of their program on the original rank list, size of resident complement, social atmosphere, leadership changes, and proximity of their training program to immediate family. Additional questions focused on mentorship and whether there was any perceived benefit to such a relationship, from the standpoint of both being a mentee and serving as a mentor to others. Specifically, mentorship was defined as "meaningful" if the trainee felt that they were benefiting from their relationship with their mentor. Finally, optional open-ended questions allowed trainees to report whether specific aspects of their training were associated with a worsened or improved training experience (Appendix Table).

Personal and professional stressors were gauged and graded along a 6-point Likert scale in terms of how residents felt they had been affected over the previous 12-24 months. Response categories were as follows: not at all or small, moderate, large, or extreme amounts. However, these categories were collapsed into a dichotomous scale for the purposes of statistical analysis, with large to extreme responses interpreted as having the variable of interest. Satisfaction in the workplace and home environments was also graded along a multipoint Likert scale from very satisfied to very dissatisfied and was similarly dichotomized for statistical interpretation. The method of converting the Likert score to a dichotomous variable, including the method of dividing groups, was based on the same methodology of conversion and group division employed by McAbee et al. in their analysis of burnout and career satisfaction in attending neurosurgeons. ${ }^{41}$ This was intended to provide a basis for direct comparison between resident and attending results. Career satisfaction was determined as the responses of "very satisfied" or "somewhat satisfied" in relation to questions addressing this pa- rameter. Variables collected in the survey included overall career satisfaction, intrinsic rewards of the profession (that is, appreciation from patients and staff), academic productivity, interpersonal relationships, and opportunities for work-life balance.

Burnout was determined according to the MBI, which uses a 7-point Likert scale from 0 through 6 to address 22 questions: $0=$ never, $1=\mathrm{a}$ few times per year, $2=$ once a month, $3=$ a few times per month, $4=$ once a week, $5=$ a few times per week, and $6=$ every day. Three subscales were used to evaluate a corresponding number of dimensions for burnout: emotional exhaustion (9 questions), depersonalization (5 questions), and low sense of personal accomplishment (8 questions). Responses were stratified into low, medium, and high categories. Consistent with previously published studies on health care workers, ${ }^{49}$ burnout was defined by high scores for emotional exhaustion $(\geq 27)$ and/or depersonalization $(\geq 10)$.

\section{Statistical Analysis}

Demographic information was compiled from a series of descriptive statistics. As previously mentioned, select survey responses using multipoint Likert scales were converted into dichotomous variables for statistical analysis. For example, when addressing career satisfaction, respondents could choose one of the following responses: "very satisfied," "somewhat satisfied," "neutral," "somewhat dissatisfied," "very dissatisfied," or "not applicable." Howev$\mathrm{er}$, in the final analyses, responses were classified as either satisfied or not satisfied. As our primary objectives were directed at burnout and career satisfaction, we performed univariate analysis to look for associations between key demographic data and clinical measures of both of these outcomes by using logistic regression. Any item found to have a $p<0.05$ on univariate testing was then placed in a multivariate analysis using a forward stepwise manner. To avoid collinearity among multiple "subjective" response collinear variables, we limited multivariate analysis to objective variables showing significance with univariate analysis and no more than 3 subjective variables. Twotailed analysis with $\mathrm{p}<0.05$ was used as the cutoff for statistical significance. Results were reported with adjusted odds ratios and corresponding $95 \%$ confidence intervals. All data were analyzed using the SAS 9.4 statistical software (SAS Institute Inc.).

\section{Results \\ Survey Results}

Of the 1643 email addresses to which the survey invitation was sent, 395 responses $(24 \%)$ were received. Of these, $346(21 \%)$ were included in the final analysis since 49 were excluded on the basis of an incomplete survey. Most residents were male (78\%), over 31 years old (52\%), in a long-term relationship (70\%), and had no children (73\%). Respondents were equally distributed across all residency years. These demographic data are summarized in Table 1.

Forty-three percent of respondents made the decision to pursue neurosurgery during years 3 and 4 of medical school, and nearly one-fifth made their choice prior 
TABLE 1. Demographic information on neurosurgery residency survey respondents

\begin{tabular}{|c|c|}
\hline Characteristic & No. $(\%)$ \\
\hline \multicolumn{2}{|l|}{ Age in yrs } \\
\hline $21-25$ & $3(1)$ \\
\hline $26-30$ & $163(47)$ \\
\hline $31-35$ & $155(45)$ \\
\hline $36-40$ & $25(7)$ \\
\hline \multicolumn{2}{|l|}{ Sex } \\
\hline Male & $270(78)$ \\
\hline Female & $76(22)$ \\
\hline \multicolumn{2}{|l|}{ Relationship status } \\
\hline Stable partner/married & $241(70)$ \\
\hline Divorced & $7(2)$ \\
\hline Single & $98(28)$ \\
\hline \multicolumn{2}{|l|}{ Children } \\
\hline No & $251(73)$ \\
\hline Yes & $95(27)$ \\
\hline \multicolumn{2}{|l|}{ No. of children } \\
\hline 0 & $251(73)$ \\
\hline 1 & $54(16)$ \\
\hline 2 & $26(8)$ \\
\hline $3+$ & $10(3)$ \\
\hline Response missing* & $5(1)$ \\
\hline \multicolumn{2}{|l|}{ PGY } \\
\hline PGY1 & $47(14)$ \\
\hline PGY2 & $50(14)$ \\
\hline PGY3 & $62(18)$ \\
\hline PGY4 & $60(17)$ \\
\hline PGY5 & $56(16)$ \\
\hline PGY6 & $47(14)$ \\
\hline PGY7 & $24(7)$ \\
\hline \multicolumn{2}{|l|}{ Program location } \\
\hline NE/NY & $45(13)$ \\
\hline Midwest & $87(25)$ \\
\hline Mid-Atlantic & $39(11)$ \\
\hline South & $90(26)$ \\
\hline Southwest & $34(10)$ \\
\hline West & $51(15)$ \\
\hline
\end{tabular}

NE/NY = Northeast/New York; PGY = postgraduate year.

* Respondent indicated that they had children but did not specify how many.

to medical school. Eighty-one percent of residents were satisfied with their career, of which $42 \%$ reported being very satisfied with their choice. Although $75 \%$ felt their professional and personal lives would improve following residency, $41 \%$ had given serious thought to quitting neurosurgery. If presented with the choice again, $79 \%$ said they would choose neurosurgery as a specialty, and $64 \%$ would recommend neurosurgery to a prospective medical school applicant. These and other perceptions are listed in Table 2.

A satisfactory work-life balance and sufficient time
TABLE 2. General perceptions among neurosurgery residents regarding their career

\begin{tabular}{ll}
\hline \multicolumn{1}{c}{ Perception } & No. (\%) \\
\hline Satisfied w/ career as neurosurgeon* & $280(81)$ \\
\hline Satisfied w/ academic productivity* & $165(47)$ \\
\hline Satisfied w/ work-life balance* & $111(32)$ \\
\hline Adequate time for personal growth/development* & $121(35)$ \\
\hline Spouse understanding of work hrs* & $241(70)$ \\
\hline Affected by control over one's schedule ${ }^{*}$ & $210(61)$ \\
\hline Would choose neurosurgery again & $274(79)$ \\
\hline Would choose their residency program again & $227(66)$ \\
\hline Would recommend neurosurgery to a prospective applicant & $222(64)$ \\
\hline Has given serious thought to quitting & $143(41)$ \\
\hline Concerned about health care reform \& future of medicine & $250(72)$ \\
\hline
\end{tabular}

* Respondents answered "very satisfied" or "somewhat satisfied."

† Respondents answered "moderate amount," "large amount," or "extreme amount."

for personal development and didactics were reported by $32 \%, 35 \%$, and $30 \%$, respectively (Fig. 1). When asked to address factors that had appreciably affected their psyche in the previous 24 months, notable stressors with at least a moderate impact included poor control over one's schedule $(61 \%)$, inadequate wages or burdensome debt (49\%), hostile faculty $(36 \%)$, hostile co-residents $(31 \%)$, and coresident attrition (31\%; Fig. 2). The majority of residents (72\%) were concerned about the direction of health care reform and how it might impact their future, with $42 \%$ describing feeling at least moderately burdened by future job prospects. When considering their quality of life as a resident, 59\% were hopeful that things would improve. Conversely, when asked if life in residency would worsen, $45 \%$ believed that was the case (Fig. 3 ).

Despite having one of the most competitive careers, $43 \%$ of residents reported a low sense of personal accomplishment (Fig. 4). High emotional exhaustion and high depersonalization rates were calculated to be $36 \%$ and $60 \%$, respectively. The overall burnout rate among neurosurgery trainees was $67 \%$. Trends of the various MBI indices by postgraduate year are listed in Table 3 and depicted in Fig. 5.

\section{Predictors of Burnout and Career Satisfaction}

Several notable demographic factors with variable levels of burnout correlation in the literature, including age, sex, postgraduate year, relationship status, and having children, ${ }^{34,35,43,55}$ were not correlated with burnout in our study. Variables with strong associations included occupational stressors, such as inadequate exposure to the operating room (OR 10.96, $\mathrm{p}<0.01$ ), hostile faculty (OR 9.02, p < 0.0001), hostile co-residents (OR 5.05, p < 0.001 ), feeling underappreciated by patients or staff (OR 5.59-7.73, $\mathrm{p}<0.0001$ ), poor control over one's schedule (OR 6.72, p < 0.0001), and co-resident attrition (OR 3.33, $\mathrm{p}<0.01)$. Results of the univariate analysis are presented in Table 4. 


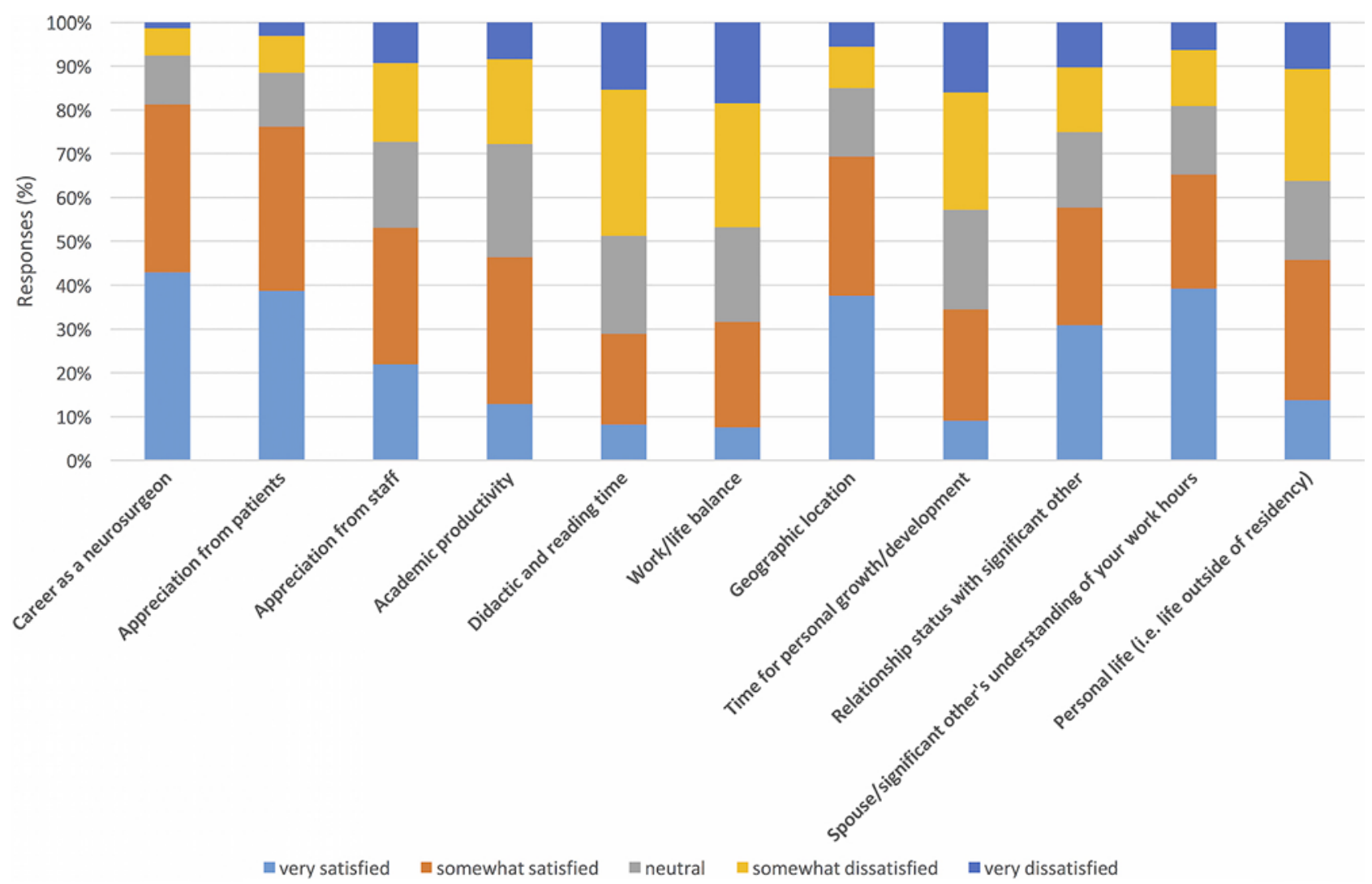

FIG. 1. Neurosurgery resident career and personal satisfaction. Figure is available in color online only.

In the multivariate analysis, notable factors associated with burnout included inadequate operating room exposure (OR 7.57, $\mathrm{p}=0.011)$, hostile faculty (OR 4.07, $\mathrm{p}=$ 0.008 ), and social stressors outside of work (OR 4.52, $\mathrm{p}=$ 0.008 ). Moreover, residents who felt they were not benefiting from their interaction with their mentors were 3 times more likely to exhibit burnout in the multivariate models (OR 2.96, $\mathrm{p}=0.031$; Table 5).

\section{Discussion}

Undue stressors in the workplace can have adverse ef-

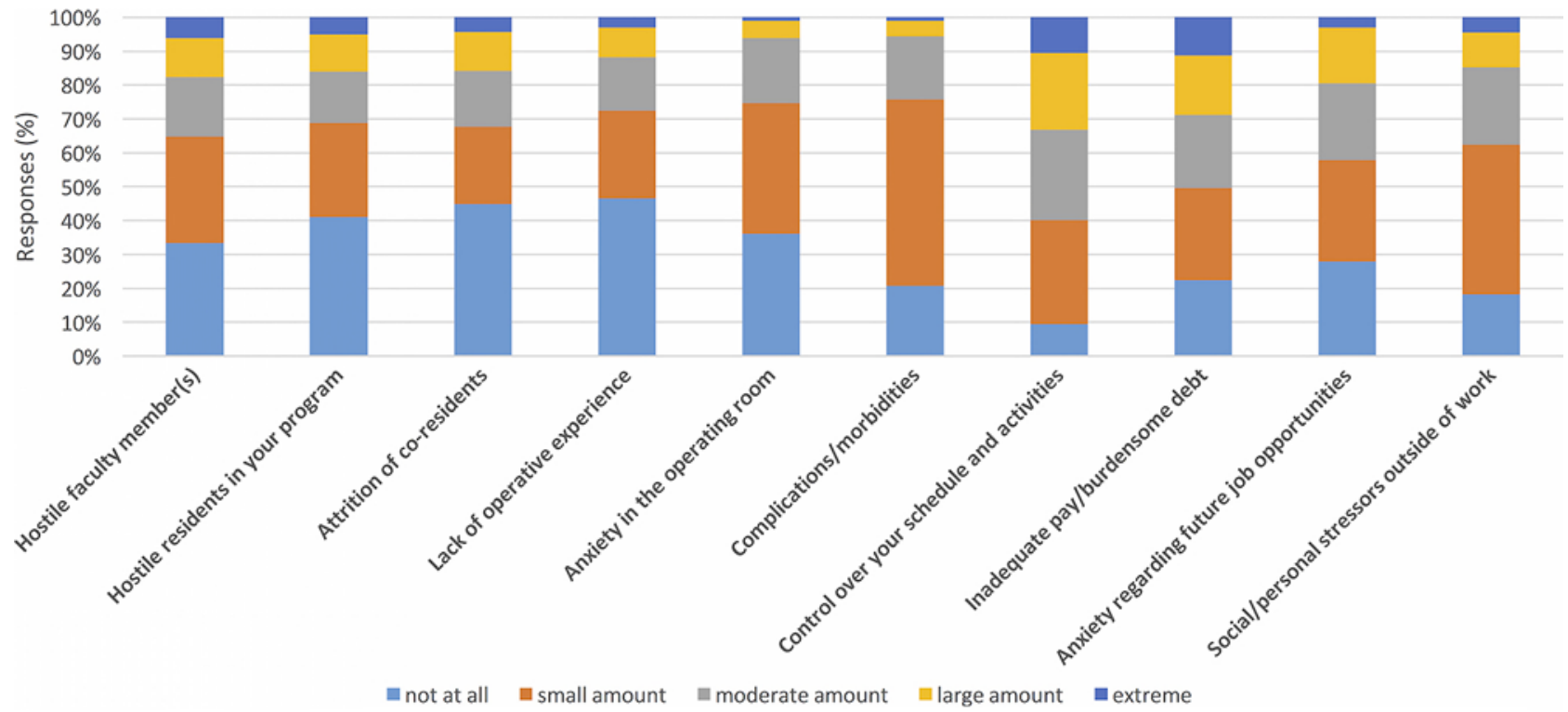

FIG. 2. Professional and personal stressors encountered by neurosurgery resident trainees. Figure is available in color online only. 
I feel that my life as a resident will improve

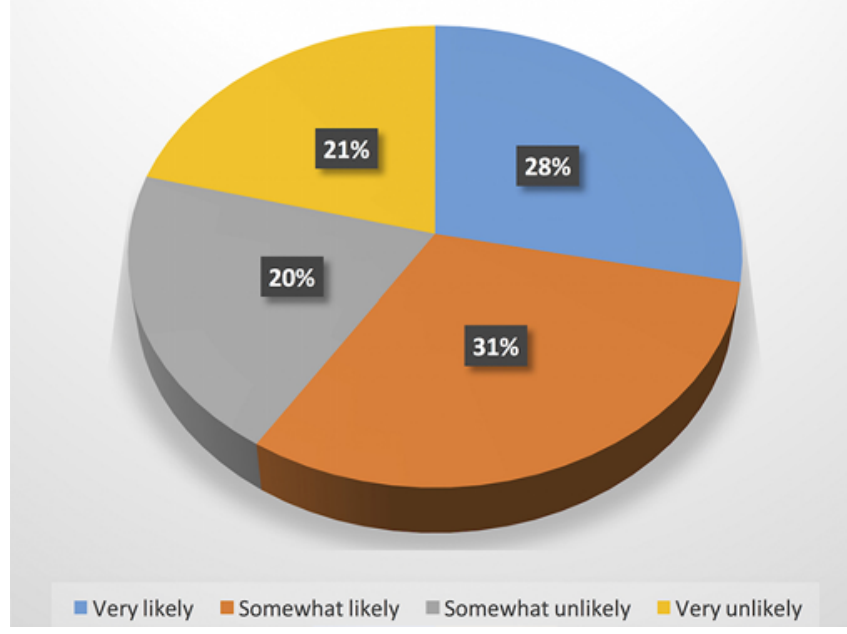

I feel that my life as a resident will worsen

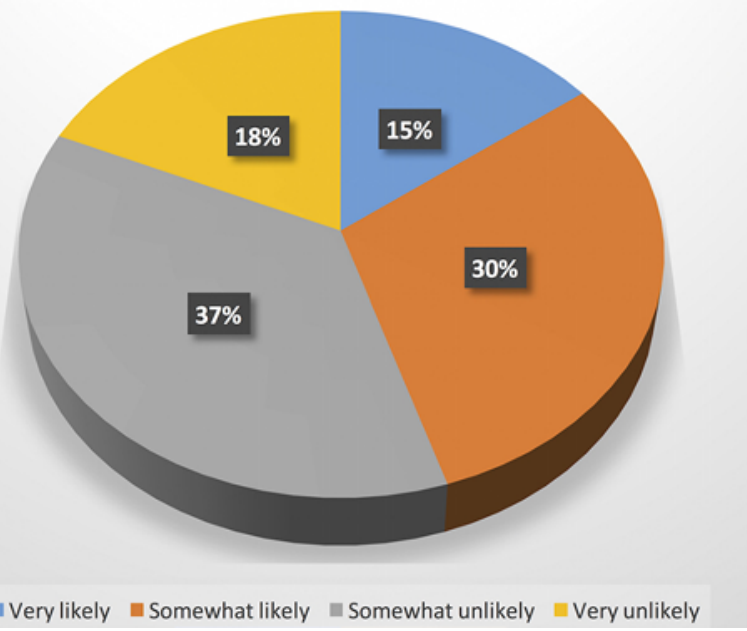

FIG. 3. Breakdown of perceptions among neurosurgery residents regarding the trajectory of their careers. Figure is available in color online only.

fects on a resident's emotional well-being with resultant detriment to patient care and health care economics. The extensive reach of burnout has prompted action because of its presumed role in medical errors and associations with higher rates of depression, substance abuse, relationship discord, and suicide. . $^{811,27,28,57,66}$ Whereas the prevalence of burnout in US workers is estimated to be $28 \%$, the number is closer to $40 \%$ for physicians and $57 \%$ for practicing neurosurgeons. ${ }^{41,56}$ Burnout is also noted to be higher in trainees than in independent practitioners of the same specialty. ${ }^{3}$ No previous studies have determined rates of burnout among neurosurgery residents. We have determined the overall rate of burnout to be $67 \%$ based on the results of a nationwide survey, one of the highest rates among residents (Table 6).

With advances in electronic communication, the line between work and home life has become increasingly blurred. Poor dissociation between work and personal life has long been recognized as a source of employee distress and burnout. Many organizations have thus devised policies limiting employee access to work matters when they walk out the door: Volkswagen turns off access to email, and Goldman Sachs and Credit Suisse have a "Saturday rule" stipulating that analysts must be away from the office for a designated period. ${ }^{29}$ Medicine followed suit in 2003, curtailing the resident workweek to 80 hours. De-

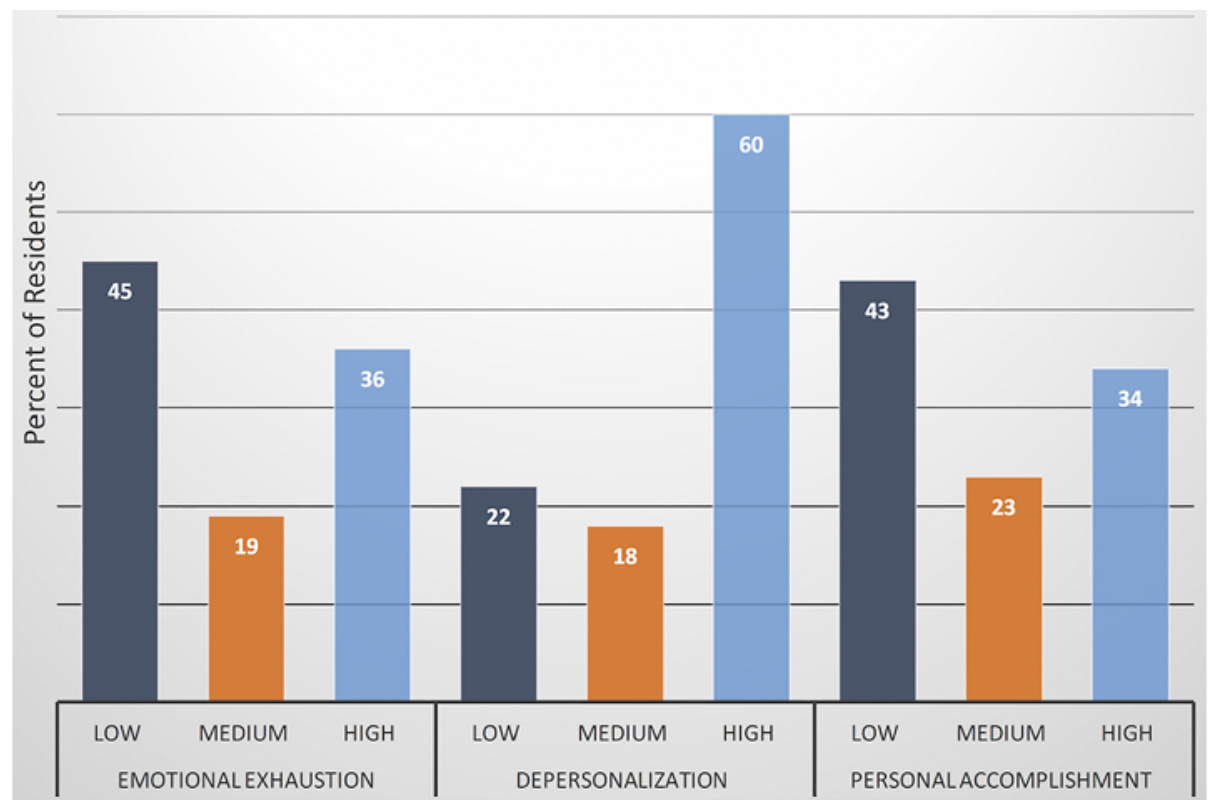

FIG. 4. Professional burnout indices among neurosurgery residents according to the MBI subscales. Figure is available in color online only. 
TABLE 3. Burnout indices among neurosurgery residents

\begin{tabular}{|c|c|c|c|c|c|c|c|}
\hline \multirow[b]{2}{*}{ Variable } & \multicolumn{7}{|c|}{ PGY } \\
\hline & 1 & 2 & 3 & 4 & 5 & 6 & 7 \\
\hline No. of residents & 47 & 50 & 62 & 60 & 56 & 47 & 24 \\
\hline \multicolumn{8}{|l|}{ Emotional exhaustion } \\
\hline Median & 17 & 24 & 24 & 20 & 23 & 17 & 15.5 \\
\hline IQR & $10-24$ & $15-37$ & $14-34$ & $11-33$ & $13-34$ & $9-26$ & $7.5-30$ \\
\hline Low (no. [\%]) & $26(55)$ & $19(38)$ & $22(35)$ & $28(47)$ & $19(34)$ & $26(55)$ & $15(63)$ \\
\hline Moderate (no. [\%]) & $11(23)$ & $8(16)$ & $14(23)$ & $9(15)$ & $12(21)$ & $10(21)$ & $2(8)$ \\
\hline High (no. [\%]) & $10(21)$ & $23(46)$ & $26(42)$ & $23(38)$ & $25(45)$ & $11(23)$ & $7(29)$ \\
\hline \multicolumn{8}{|l|}{ Depersonalization } \\
\hline Median & 10 & 13 & 12.5 & 12 & 11.5 & 8 & 10 \\
\hline IQR & $4-15$ & $7-19$ & $8-17$ & $7-17$ & $7-15.5$ & $4-14$ & $3.5-14.5$ \\
\hline Low (no. [\%]) & $15(32)$ & $9(18)$ & $10(16)$ & $9(15)$ & $11(20)$ & $14(30)$ & $7(29)$ \\
\hline Moderate (no. [\%]) & $6(13)$ & $8(16)$ & $9(15)$ & $12(20)$ & $11(20)$ & $13(28)$ & $5(21)$ \\
\hline High (no. [\%]) & $26(55)$ & $33(66)$ & $43(69)$ & $39(65)$ & $34(61)$ & $20(43)$ & $12(50)$ \\
\hline \multicolumn{8}{|c|}{ Personal accomplishment } \\
\hline Median & 36 & 36 & 32.5 & 34.5 & 35 & 40 & 38 \\
\hline IQR & $30-42$ & $28-41$ & $28-41$ & $28.5-40$ & $26.5-42$ & $32-44$ & $29.5-44$ \\
\hline Low (no. [\%]) & $21(45)$ & $22(44)$ & $33(53)$ & $27(45)$ & $24(43)$ & $13(28)$ & $9(38)$ \\
\hline Moderate (no. [\%]) & $11(23)$ & $12(24)$ & $11(18)$ & $15(25)$ & $15(27)$ & $10(21)$ & $4(17)$ \\
\hline High (no. [\%]) & $15(32)$ & $16(32)$ & $18(29)$ & $18(30)$ & $17(30)$ & $24(51)$ & $11(46)$ \\
\hline
\end{tabular}

$\mathrm{IQR}=$ interquartile range.

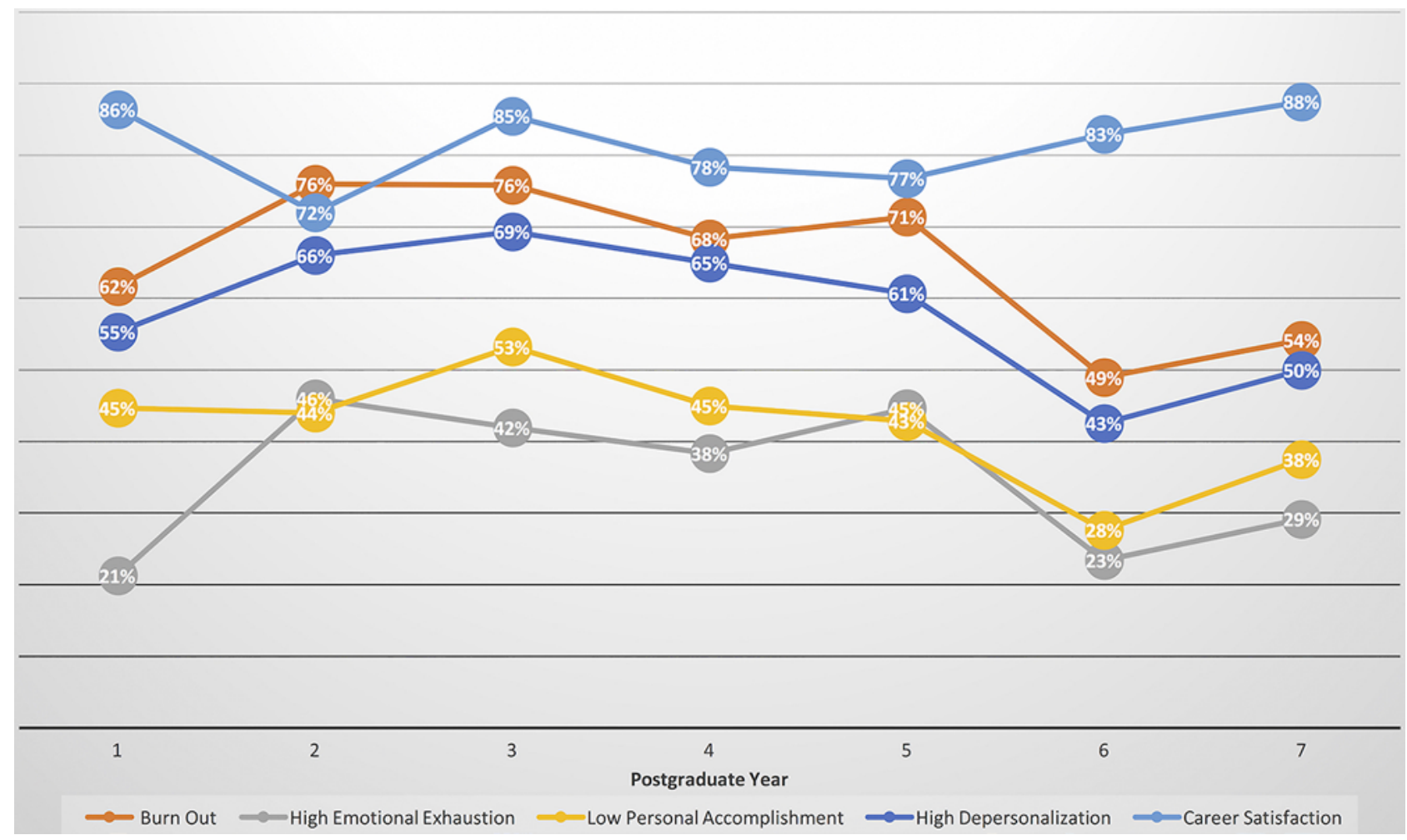

FIG. 5. Maslach Burnout Inventory and career satisfaction trends by postgraduate year. Figure is available in color online only. 
TABLE 4. Univariate analysis of burnout and career satisfaction among neurosurgery residents

\begin{tabular}{|c|c|c|c|c|c|c|c|c|}
\hline \multirow[b]{2}{*}{ Variable } & \multicolumn{4}{|c|}{ Burnout } & \multicolumn{4}{|c|}{ Career Satisfaction } \\
\hline & OR & \multicolumn{2}{|c|}{$95 \% \mathrm{Cl}$} & $p$ Value & OR & \multicolumn{2}{|c|}{$95 \% \mathrm{Cl}$} & $p$ Value \\
\hline Sex & & & & & & & & \\
\hline Male & 0.72 & 0.41 & 1.25 & 0.24 & 1.45 & 0.78 & 2.68 & 0.24 \\
\hline Female & \multicolumn{4}{|c|}{ Reference } & \multicolumn{4}{|c|}{ Reference } \\
\hline \multicolumn{9}{|l|}{ Relationship status } \\
\hline Stable partner/married & \multicolumn{4}{|c|}{ Reference } & \multicolumn{4}{|c|}{ Reference } \\
\hline Divorced & 3.39 & 0.40 & 28.61 & 0.26 & 0.28 & 0.06 & 1.28 & 0.10 \\
\hline Single & 1.49 & 0.89 & 2.49 & 0.13 & 0.71 & 0.39 & 1.26 & 0.24 \\
\hline \multicolumn{9}{|l|}{ Children } \\
\hline No & 1.51 & 0.92 & 2.46 & 0.10 & 1.07 & 0.59 & 1.94 & 0.83 \\
\hline Yes & \multicolumn{4}{|c|}{ Reference } & \multicolumn{4}{|c|}{ Reference } \\
\hline \multicolumn{9}{|l|}{ PGY } \\
\hline PGY1 & \multicolumn{4}{|c|}{ Reference } & \multicolumn{4}{|c|}{ Reference } \\
\hline PGY2 & 1.97 & 0.82 & 4.72 & 0.13 & 0.41 & 0.14 & 1.17 & 0.10 \\
\hline PGY3 & 1.95 & 0.85 & 4.45 & 0.11 & 0.93 & 0.31 & 2.83 & 0.90 \\
\hline PGY4 & 1.34 & 0.60 & 2.98 & 0.47 & 0.57 & 0.20 & 1.64 & 0.30 \\
\hline PGY5 & 1.55 & 0.68 & 3.54 & 0.30 & 0.52 & 0.18 & 1.51 & 0.23 \\
\hline PGY6 & 0.60 & 0.26 & 1.35 & 0.21 & 0.77 & 0.24 & 2.43 & 0.66 \\
\hline PGY7 & 0.73 & 0.27 & 1.99 & 0.54 & 1.11 & 0.25 & 4.88 & 0.89 \\
\hline \multicolumn{9}{|l|}{ Would you choose neurosurgery again? } \\
\hline No & 5.31 & 2.42 & 11.64 & $<0.0001$ & 0.05 & 0.02 & 0.11 & $<0.0001$ \\
\hline Yes & \multicolumn{4}{|c|}{ Reference } & \multicolumn{4}{|c|}{ Reference } \\
\hline Don't know & 2.80 & 1.37 & 5.72 & $<0.01$ & 0.12 & 0.06 & 0.25 & $<0.0001$ \\
\hline \multicolumn{9}{|c|}{ Would you choose your residency training program again? } \\
\hline No & 5.31 & 2.42 & 11.64 & $<0.0001$ & 0.12 & 0.06 & 0.24 & $<0.0001$ \\
\hline Yes & \multicolumn{4}{|c|}{ Reference } & & & Ference & \\
\hline Don't know & 2.80 & 1.37 & 5.72 & $<0.0001$ & 0.25 & 0.12 & 0.54 & $<0.0001$ \\
\hline Would you recommend neurosurgery to a medi & & & & & & & & \\
\hline No & 10.65 & 3.74 & 30.35 & $<0.0001$ & 0.13 & 0.07 & 0.25 & $<0.0001$ \\
\hline Yes & & Ref & erence & & & & ference & \\
\hline Don't know & 1.94 & 1.05 & 3.61 & 0.04 & 0.35 & 0.17 & 0.73 & $<0.0001$ \\
\hline How likely do you feel that your life as a resider & & & & & & & & \\
\hline Unlikely & 1.93 & 1.20 & 3.09 & $<0.01$ & 0.29 & 0.17 & 0.51 & $<0.0001$ \\
\hline Likely & & Ref & erence & & & & ference & \\
\hline How likely do you feel your life as a resident wil & & & & & & & & \\
\hline Unlikely & 0.39 & 0.24 & 0.62 & $<0.0001$ & 3.06 & 1.75 & 5.37 & $<0.0001$ \\
\hline Likely & & Ref & erence & & & & Frence & \\
\hline At some point during residency, have you given & & & & & & & & \\
\hline No & 0.22 & 0.13 & 0.37 & $<0.0001$ & 5.61 & 3.06 & 10.28 & $<0.0001$ \\
\hline Yes & & Ref & erence & & & & Erence & \\
\hline $\begin{array}{l}\text { Are you or do you intend to get significantly invo } \\
\text { surgical societies, such as AANS or CNS? }\end{array}$ & & & & & & & & \\
\hline No & 1.75 & 1.04 & 2.92 & 0.03 & 0.35 & 0.20 & 0.60 & $<0.001$ \\
\hline Yes & & Ref & erence & & & & Ference & \\
\hline Do you feel you had an accurate perception of & & & & & & & & \\
\hline No & 3.15 & 1.82 & 5.45 & $<0.0001$ & 0.37 & 0.21 & 0.66 & $<0.001$ \\
\hline Yes & & Ref & erence & & & $\operatorname{Re}$ & Ference & \\
\hline
\end{tabular}


» CONTINUED FROM PAGE 1356

TABLE 4. Univariate analysis of burnout and career satisfaction among neurosurgery residents

\begin{tabular}{|c|c|c|c|c|c|c|c|c|}
\hline \multirow[b]{2}{*}{ Variable } & \multicolumn{4}{|c|}{ Burnout } & \multicolumn{4}{|c|}{ Career Satisfaction } \\
\hline & OR & $95 \%$ & $\% \mathrm{Cl}$ & p Value & OR & $95 \%$ & $\% \mathrm{Cl}$ & $p$ Value \\
\hline \multicolumn{9}{|c|}{ Do you socialize w/ your co-residents outside of work? } \\
\hline No & 2.75 & 1.34 & 5.67 & $<0.01$ & 0.41 & 0.22 & 0.76 & $<0.01$ \\
\hline Yes & \multicolumn{4}{|c|}{ Reference } & \multicolumn{4}{|c|}{ Reference } \\
\hline \multicolumn{9}{|c|}{ Do you feel you are performing adequately for your stage of training? } \\
\hline No & 4.63 & 1.60 & 13.40 & $<0.01$ & 0.22 & 0.11 & 0.46 & $<0.0001$ \\
\hline Yes & \multicolumn{4}{|c|}{ Reference } & \multicolumn{4}{|c|}{ Reference } \\
\hline \multicolumn{9}{|c|}{$\begin{array}{l}\text { Do you think your professional \& personal life will significantly improve AFTER } \\
\text { residency? }\end{array}$} \\
\hline No & 1.60 & 0.62 & 4.15 & 0.33 & 0.21 & 0.09 & 0.51 & $<0.001$ \\
\hline Yes & \multicolumn{4}{|c|}{ Reference } & \multicolumn{4}{|c|}{ Reference } \\
\hline \multicolumn{9}{|c|}{ Do you have a mentor (informal or formally assigned)? } \\
\hline No & 1.51 & 0.92 & 2.50 & 0.11 & 0.67 & 0.38 & 1.17 & 0.16 \\
\hline Yes & \multicolumn{4}{|c|}{ Reference } & \multicolumn{4}{|c|}{ Reference } \\
\hline \multicolumn{9}{|c|}{ Do you feel you are benefiting from your relationship w/ your mentor? } \\
\hline No & 4.12 & 1.85 & 9.19 & $<0.001$ & 0.23 & 0.11 & 0.45 & $<0.0001$ \\
\hline Yes & \multicolumn{4}{|c|}{ Reference } & \multicolumn{4}{|c|}{ Reference } \\
\hline Not applicable & 2.33 & 1.34 & 4.06 & $<0.01$ & 0.42 & 0.22 & 0.80 & $<0.01$ \\
\hline \multicolumn{9}{|c|}{ Do you act as a mentor for other residents or students? } \\
\hline No & 1.63 & 0.97 & 2.75 & 0.07 & 0.43 & 0.25 & 0.76 & $<0.01$ \\
\hline Yes & \multicolumn{4}{|c|}{ Reference } & \multicolumn{4}{|c|}{ Reference } \\
\hline \multicolumn{9}{|l|}{ In the past $12-24$ mos, have you felt the following? } \\
\hline Hostile faculty member(s) & & & & & & & & \\
\hline Large to extreme & 9.02 & 3.18 & 25.56 & $<0.0001$ & 0.23 & 0.13 & 0.43 & $<0.0001$ \\
\hline None to moderate & & Refe & erence & & & Ref & ference & \\
\hline Hostile residents in your program & & & & & & & & \\
\hline Large to extreme & 5.05 & 2.10 & 12.17 & $<0.001$ & 0.49 & 0.26 & 0.93 & 0.03 \\
\hline None to moderate & & Refe & erence & & & Ref & ference & \\
\hline Attrition of co-residents & & & & & & & & \\
\hline Large to extreme & 3.33 & 1.51 & 7.34 & $<0.01$ & 0.49 & 0.25 & 0.95 & 0.03 \\
\hline None to moderate & & Refe & erence & & & Ref & ference & \\
\hline Lack of op experience & & & & & & & & \\
\hline Large to extreme & 10.96 & 2.59 & 46.36 & $<0.01$ & 0.37 & 0.18 & 0.76 & $<0.01$ \\
\hline None to moderate & & Refe & erence & & & Ref & ference & \\
\hline Lack of control over your schedule \& activitie & & & & & & & & \\
\hline Large to extreme & 6.72 & 3.50 & 12.90 & $<0.0001$ & 0.28 & 0.16 & 0.49 & $<0.0001$ \\
\hline None to moderate & & Refe & erence & & & Ref & ference & \\
\hline Inadequate pay/burdensome debt & & & & & & & & \\
\hline Large to extreme & 1.78 & 1.05 & 3.02 & 0.03 & 0.45 & 0.26 & 0.78 & $<0.01$ \\
\hline None to moderate & & Refe & erence & & & Ref & ference & \\
\hline Anxiety regarding future job opportunities & & & & & & & & \\
\hline Large to extreme & 1.81 & 0.98 & 3.34 & 0.06 & 0.22 & 0.12 & 0.40 & $<0.0001$ \\
\hline None to moderate & & Refe & erence & & & Ref & ference & \\
\hline Social/personal stressors outside of work & & & & & & & & \\
\hline Large to extreme & 4.32 & 1.78 & 10.45 & $<0.01$ & 0.29 & 0.15 & 0.55 & $<0.001$ \\
\hline None to moderate & & Refe & erence & & & Ref & ference & \\
\hline
\end{tabular}


» CONTINUED FROM PAGE 1357

TABLE 4. Univariate analysis of burnout and career satisfaction among neurosurgery residents

\begin{tabular}{|c|c|c|c|c|c|c|c|c|}
\hline \multirow[b]{2}{*}{ Variable } & \multicolumn{4}{|c|}{ Burnout } & \multicolumn{4}{|c|}{ Career Satisfaction } \\
\hline & OR & 95 & $6 \mathrm{Cl}$ & p Value & OR & $95^{\circ}$ & $\% \mathrm{Cl}$ & $\mathrm{p}$ Value \\
\hline \multicolumn{9}{|c|}{ In relation to your residency, how satisfied are you w/ the following factors? } \\
\hline \multicolumn{9}{|l|}{ Career as a neurosurgeon } \\
\hline Not satisfied & 13.63 & 4.17 & 44.48 & $<0.0001$ & & & & \\
\hline Satisfied & \multicolumn{4}{|c|}{ Reference } & & & & \\
\hline \multicolumn{9}{|l|}{ Appreciation from patients } \\
\hline Not satisfied & 5.59 & 2.68 & 11.66 & $<0.0001$ & 0.15 & 0.08 & 0.26 & $<0.0001$ \\
\hline Satisfied & \multicolumn{4}{|c|}{ Reference } & \multicolumn{4}{|c|}{ Reference } \\
\hline \multicolumn{9}{|l|}{ Appreciation from staff } \\
\hline Not satisfied & 7.73 & 4.45 & 13.43 & $<0.0001$ & 0.19 & 0.10 & 0.36 & $<0.0001$ \\
\hline Satisfied & \multicolumn{4}{|c|}{ Reference } & \multicolumn{4}{|c|}{ Reference } \\
\hline \multicolumn{9}{|l|}{ Academic productivity } \\
\hline Not satisfied & 3.08 & 1.92 & 4.93 & $<0.0001$ & 0.13 & 0.06 & 0.27 & $<0.0001$ \\
\hline Satisfied & \multicolumn{4}{|c|}{ Reference } & \multicolumn{4}{|c|}{ Reference } \\
\hline \multicolumn{9}{|l|}{ Didactic \& reading time } \\
\hline Not satisfied & 3.04 & 1.87 & 4.94 & $<0.0001$ & 0.12 & 0.04 & 0.35 & $<0.0001$ \\
\hline Satisfied & \multicolumn{4}{|c|}{ Reference } & \multicolumn{4}{|c|}{ Reference } \\
\hline \multicolumn{9}{|l|}{ Work-life balance } \\
\hline Not satisfied & 3.94 & 2.43 & 6.40 & $<0.0001$ & 0.11 & 0.04 & 0.31 & $<0.0001$ \\
\hline Satisfied & \multicolumn{4}{|c|}{ Reference } & \multicolumn{4}{|c|}{ Reference } \\
\hline \multicolumn{9}{|l|}{ Geographic location } \\
\hline Not satisfied & 2.26 & 1.33 & 3.84 & $<0.01$ & 0.31 & 0.18 & 0.54 & $<0.0001$ \\
\hline Satisfied & \multicolumn{4}{|c|}{ Reference } & \multicolumn{4}{|c|}{ Reference } \\
\hline \multicolumn{9}{|l|}{ Time for personal growth/development } \\
\hline Not satisfied & 4.37 & 2.70 & 7.08 & $<0.0001$ & 0.18 & 0.08 & 0.41 & $<0.0001$ \\
\hline Satisfied & & Ref & erence & & & & erence & \\
\hline Relationship status w/ significant other & & & & & & & & \\
\hline Not satisfied & 2.10 & 1.28 & 3.44 & $<0.01$ & 0.32 & 0.18 & 0.57 & $<0.0001$ \\
\hline Satisfied & & Ref & erence & & & $\operatorname{Re}$ & erence & \\
\hline Spouse/significant other's understanding of & & & & & & & & \\
\hline Not satisfied & 1.79 & 1.05 & 3.04 & 0.03 & 0.33 & 0.19 & 0.60 & $<0.0001$ \\
\hline Satisfied & & Ref & erence & & & & erence & \\
\hline Personal life (i.e., life outside of residency) & & & & & & & & \\
\hline Not satisfied & 2.80 & 1.76 & 4.45 & $<0.0001$ & 0.28 & 0.15 & 0.53 & $<0.0001$ \\
\hline Satisfied & & Ref & erence & & & $\mathrm{Re}$ & erence & \\
\hline Exercise & & & & & & & & \\
\hline No & & Ref & erence & & & $\mathrm{Re}$ & erence & \\
\hline Yes & 0.57 & 0.34 & 0.94 & 0.03 & 1.59 & 0.91 & 2.78 & 0.10 \\
\hline
\end{tabular}

spite this change, $65 \%$ of neurosurgery residents reported insufficient time for personal growth and development and $68 \%$ had not achieved a satisfactory work-life balance. These individuals were, in turn, 4 times more likely to suffer burnout (OR 4.37, $\mathrm{p}<0.0001)$, according to univariate analysis. This is not surprising, as time outside the hospital is often filled with scholarly pursuits, including independent study, operative planning, and research. Therefore, successfully combating burnout and improving resident quality of life extends beyond work hours. A recent pi- lot study addressed neurosurgery departmental wellness through a physical education and nutrition program, promising steps in managing stress. ${ }^{61}$

Central to physician wellness is the intrinsic reward and career satisfaction offered by the specialty. While neurosurgery is renowned for arduous training, its tremendous impact makes for thankful patients and a rewarding profession. This may, in part, explain why traditionally challenging fields with high susceptibility to burnout can also have high satisfaction rates. However, a decrease in intrin- 
TABLE 5. Multivariable logistic regression analysis of burnout

\begin{tabular}{|c|c|c|c|}
\hline \multirow[b]{2}{*}{ Variable } & \multicolumn{3}{|c|}{ Burnout } \\
\hline & OR & $95 \% \mathrm{Cl}$ & p Value \\
\hline \multicolumn{4}{|c|}{ Would you recommend neurosurgery to a medical student? } \\
\hline No & 6.40 & $1.85,22.08$ & 0.003 \\
\hline Yes & \multicolumn{3}{|c|}{ Reference } \\
\hline \multicolumn{4}{|c|}{ When considering quitting, would you have left the medical field? } \\
\hline No & 3.63 & $0.69,19.20$ & 0.129 \\
\hline Yes & \multicolumn{3}{|c|}{ Reference } \\
\hline \multicolumn{4}{|c|}{ Do you feel you are benefiting from your relationship w/ your mentor? } \\
\hline No & 2.96 & $1.11,7.90$ & 0.031 \\
\hline Yes & \multicolumn{3}{|c|}{ Reference } \\
\hline Not applicable & 1.51 & $0.75,3.06$ & 0.251 \\
\hline \multicolumn{4}{|l|}{ No. of children } \\
\hline 0 & \multicolumn{3}{|c|}{ Reference } \\
\hline 1 & 0.55 & $0.24,1.22$ & 0.14 \\
\hline 2 & 0.35 & $0.13,0.98$ & 0.046 \\
\hline 3 & 8.47 & $0.91,78.98$ & 0.061 \\
\hline $4+$ & \multicolumn{3}{|c|}{ NA } \\
\hline \multicolumn{4}{|l|}{ Lack of op experience } \\
\hline None to moderate & \multicolumn{3}{|c|}{ Reference } \\
\hline Large to extreme & 7.57 & $1.59,35.95$ & 0.011 \\
\hline \multicolumn{4}{|l|}{ Social/personal stressors outside of work } \\
\hline None to moderate & \multicolumn{3}{|c|}{ Reference } \\
\hline Large to extreme & 4.52 & $1.47,13.84$ & 0.008 \\
\hline \multicolumn{4}{|l|}{ Difficult/hostile faculty member } \\
\hline None to moderate & \multicolumn{3}{|c|}{ Reference } \\
\hline Large to extreme & 4.07 & $1.44,11.47$ & 0.008 \\
\hline
\end{tabular}

$\mathrm{NA}=$ not applicable.

sic reward is often followed by a corresponding decrease in satisfaction. Residents who felt underappreciated by patients or staff had a nearly 7-fold reduction in career satisfaction (OR 0.15 and 0.19 , respectively, $\mathrm{p}<0.0001)$ and an 8 -fold increase in burnout (OR 5.59 and 7.73, respectively, $\mathrm{p}<0.0001$ ). Despite the high burnout rates, $81 \%$ described being satisfied with their career, and $79 \%$ would pursue neurosurgery as a specialty again if given the choice. This paradoxical relationship between burnout and career satisfaction has been noted in the literature and is often attributed to the subjectivity inherent to burnout surveys and the episodic nature of burnout over the course of training. 5,38,41

A review of the outcome trends revealed that a high degree of career satisfaction was maintained throughout all 7 years of training (Fig. 5). However, burnout rates spiked in postgraduate years 2 and $3(76 \%)$, trending downward thereafter. These findings are possibly attributable to the change in clinical responsibility and shared decision making, which is greatest across these years when one stands on the front lines as the "consultant" resident. While one cannot generalize the natural course of burnout between specialties, it is curious that an analogous upswing in burnout occurs in internal medicine and pediatrics, with the trend reversing 2 years into training., ${ }^{7,46,65}$ This reversal may result from the proverbial "light at the end of the tun- nel" as residency nears completion. Nonetheless, these patterns may offer insight into the timing of any intervention targeting burnout, as well as those persons most likely to benefit from therapy.

In recent years, pursuing a career in medicine has become synonymous with being saddled with high financial debt. The median indebtedness of medical school graduates in 1992 stood at just around $\$ 50,000 . .^{53}$ By 2016, this figure approached $\$ 190,000 .{ }^{4}$ And yet, resident wages have remained relatively stagnant over this period. While the decision to pursue a specific specialty is likely multifactorial, a growing body of evidence implicates debt and lifestyle as major determinants of career choice. ${ }^{16}$ Some studies indicate, intuitively so, that graduates with higher debt are more likely to pursue higher-paid specialties. ${ }^{32,63}$ In a longitudinal study of students from 2 prominent medical schools over an 8-year period, Grayson et al. discovered that higher debt loads were predictive of a student's likelihood to switch career trajectories from primary care to a higher-paying alternative. ${ }^{32}$ Because debt influences the timing of major life events, such as the decision to start a family or buy a home,,$^{53}$ it may ultimately serve as a source of stress and burnout in those who may be forced to postpone or forego these milestones. Indeed, neurosurgery residents from our survey with burdensome debt were twice 
TABLE 6. Published burnout rates for various residency specialties

\begin{tabular}{|c|c|c|c|c|}
\hline Authors \& Year & Residency & $\begin{array}{l}\text { No. of Respondents } \\
\text { (completion rate) }\end{array}$ & $\begin{array}{c}\text { Burnout } \\
\text { Rate }^{*}\end{array}$ & Comment \\
\hline West et al., 2011 & IM & $16,394(74 \%)$ & $51.5 \%$ & $\begin{array}{l}\mathrm{MBI} \text {; national survey of all IM residents in US w/ } \\
\text { data collected at time of annual in-training exam }\end{array}$ \\
\hline Shanafelt et al., 2002 & IM & $115(76 \%)$ & $76 \%$ & $\mathrm{MBl}$; single-institution study in US \\
\hline Fahrenkopf et al., 2008 & Pediatrics & $123(100 \%) \dagger$ & $74 \%$ & MBI; multicenter prospective study in US \\
\hline Pantaleoni et al., 2014 & Pediatrics & $\begin{array}{l}\text { Varied throughout study } \\
\text { from } 54 \% \text { to } 100 \% \text {; larg- } \\
\text { est cohort } 80 \text { residents }\end{array}$ & $\begin{array}{l}17 \% \text { (intern yr), } \\
40 \% \text { (junior yr), } \\
42 \% \text { (senior yr) }\end{array}$ & $\begin{array}{l}\text { MBI; longitudinal cohort study across multiple } \\
\text { centers }\end{array}$ \\
\hline Kimo Takayesu et al., 2014 & EM & $218(67 \%)$ & $65 \%$ & $\begin{array}{l}\text { MBI; multi-institutional study across } 8 \text { EM programs } \\
\text { in US }\end{array}$ \\
\hline Nyssen et al., 2003 & Anesthesiology & $151(48 \%)$ & $40.4 \%$ & $\begin{array}{l}\mathrm{MBI} \text {; anesthetists in training \& independent practi- } \\
\text { tioners surveyed w/in Belgium university network }\end{array}$ \\
\hline Elmore et al., 2016 & General surgery & $753(\mathrm{NK})$ & $69 \%$ & MBI; national survey in US \\
\hline Golub et al., 2007 & ENT & $684(50 \%)$ & $86 \%$ & $\begin{array}{l}\text { MBI; burnout subdivided into moderate \& high } \\
\text { subscores; } 76 \% \text { exhibited the former \& } 10 \% \text { the } \\
\text { latter; national survey of ENT residents in US }\end{array}$ \\
\hline Aldrees et al., 2015 & ENT & $85(69 \%)$ & $45 \%$ & MBI; multicenter study across Saudi Arabia \\
\hline Chaput et al., 2015 & Plastic surgery & $52(61 \%)$ & $29 \%$ & $\mathrm{MBI}$; national survey of residents in France \\
\hline Arora et al., 2014 & Orthopedic surgery & $236(22 \%)$ & $53 \%$ & $\mathrm{MBI}$; national survey of Australian trainees \\
\hline Becker et al., 2006 & Ob/gyn & $125(29 \%)$ & $83 \%$ & $\begin{array}{l}\text { MBI; moderate burnout reported; multicenter study } \\
\text { across US }\end{array}$ \\
\hline Castelo-Branco et al., 2007 & Ob/gyn & $109(67 \%)$ & $58 \%$ & MBI; multicenter study across Spain \\
\hline
\end{tabular}

EM = emergency medicine; ENT = ear, nose, and throat; IM = internal medicine; NK = not known; ob/gyn = obstetrics and gynecology.

* Definitions of burnout were not consistent across studies.

$\dagger$ Prospective cohort study.

as likely to exhibit burnout (OR $1.78, \mathrm{p}=0.03$ ) with a corresponding reduction in career satisfaction (OR 0.45, p < 0.01 ), according to univariate analysis.

Medical school graduates have regarded mentorship as the single most influential factor on specialty choice. ${ }^{44}$ It has also been viewed as an important catalyst for success in residency training and beyond. For instance, increased academic productivity based on higher numbers of publications and grants, , ${ }_{1,52,62}$ a greater likelihood for promotion, ${ }^{70}$ and higher rates of career satisfaction ${ }^{45,54}$ have all been directly linked to mentorship. Despite these benefits, less than $50 \%$ of medical students, residents, and junior faculty report having mentors. ${ }^{1,33,50}$ The problem seems less to do with the desire for mentorship and more to do with excessive clinical, administrative, and scholarly demands on faculty that preclude time for meaningful mentor-mentee relationships. ${ }^{14,47}$ Sixty-eight percent of respondents in our survey claimed to have a mentor of some sort, which is higher than rates in the literature. Among those with a mentor, approximately $20 \%$ described feeling that they were not significantly benefiting from the interaction, and they, in turn, exhibited 3 -fold more burnout (OR $2.96, p=0.03$ ), according to multivariable analysis. Respondents were further offered the opportunity to expand on their mentor relationships, with common responses that they valued "career guidance," "encouragement from staff," and "faculty research guidance/productivity." This suggests that, ultimately, it is the quality rather than the nominal nature of the mentoring relationship that matters.
For mentoring to be effective, mentors may need to undergo development training. In one survey, even those with significant mentorship experience expressed a desire for training workshops that addressed complex issues such as race and culture in the workplace. ${ }^{64}$ Increased availability of career development sessions in which prospective mentors could be formally trained in the art of mentorship may be a potentially useful adjunct for faculty at training hospitals. Such an addition would also likely be self-perpetuating since those with effective mentors are generally more likely to offer mentorship to others. ${ }^{62}$

Graded responsibility and supervision through a hierarchical structure is a cornerstone of surgical education. ${ }^{47}$ Much has changed since the inception of this Halstedian tradition in 1889-notably, a cap on excessive hours and an erosion of the supervised independence residents once enjoyed. However, many traditions persist within the hierarchy, including a carryover of the mentality to "do unto others as you would have others do unto you." Almost a third of residents surveyed in our study noted that their lives entailed dealing with hostile faculty or residents within the past 2 years. The presence of difficult interactions with faculty increased burnout more than 4-fold (OR 4.07, $\mathrm{p}=$ 0.008 ), according to multivariate analysis. While the true nature of these interactions cannot be further investigated, relationships between trainees and their senior residents and faculty can often be strained. Hostile encounters and other negative experiences are known deterrents to pursuing surgery. ${ }^{33}$ In light of the precipitous decline in medi- 
cal students' interest in surgery compared with that in the $1980 \mathrm{~s},{ }^{48}$ hostile practices must be further addressed by programs to successfully combat burnout and preserve surgical pedigree.

Meaningful mentorship and personal stressors were significant predictors of burnout and career satisfaction in a multivariable regression model. However, high degrees of correlation between individual subjective predictor variables, such as positive responses to multiple stressors occurring together, limited the ability to include all answers to purely subjective questions in the multivariate analysis. The use of subjective rating scales in our questionnaire and data analysis limits objective interpretation of the impact of individual subjective factors, though we did attempt to mitigate this through limited incorporation of subjective variables in our multivariate model and by modeling our survey off of prior neurosurgical burnout studies in the attending population. Current limitations of this work are those typical of surveys, such as inaccurate reporting by respondents due to recall bias. Our response rate was only $21 \%$, which is on the lower end of the spectrum compared with rates in other published studies on trainees. Thus, one possible scenario is a skewing of data trends secondary to disproportionate completion by a particular subgroup (that is, so-called response bias). This may be due to the fact that those who suffer burnout are either less likely to complete the survey because of apathy or more likely to do so because of interest in the subject matter. In addition, despite a rigorous de-identification process for respondents, it is unclear whether our response rate was affected by potential concerns regarding confidentiality of responses. Despite the relatively low response rate, our data showed a relatively homogeneous distribution of responses, with approximately one-quarter of the responses corresponding to the East Coast, the West Coast, the South, and the Midwest. In addition, responses were similarly distributed at $14 \%-18 \%$ per year among the postgraduate years. As the direction of influence cannot be ascertained, there may be some degree of response bias that remains unaccounted for. Future studies could include a repeat of the above with greater incentives to enhance completion rates. Additionally, because each respondent was linked to a unique identifier, it would be informative to track resident responses over the course of their residency to more accurately delineate burnout trends and identify causal factors.

\section{Conclusions}

Burnout is a pervasive problem among both physicians in practice and residents in training. Several factors were independently identified as predictors of burnout, including personal and occupational stressors, which impact resident education and probably patient-related outcomes. Analysis of burnout trends revealed a spike between postgraduate years 1 and 2, suggesting the best timing for any intervention. Mentorship was identified for its putative benefit in combating burnout and may be one systematic approach that programs can formally adopt. Despite work-hour restrictions, both poor work-life dissociation and limited time for personal growth and development remain central issues. Nonetheless, the majority of residents were satis- fied with their professional careers, were hopeful about the future of health care, and would choose neurosurgery as a profession again if given the choice.

\section{Acknowledgments}

We acknowledge Katherine Guzman of the University of Southern California neurosurgery program, as well as national neurosurgery program coordinators, for the organization, counsel, and promotion of the national effort to assemble and disseminate our burnout survey. We also acknowledge Sandy Meyer, administrator for the Council of State Neurosurgical Societies, for her assistance with project organization, access to SurveyMonkey, and anonymous dissemination of the survey to the resident list.

\section{References}

1. Aagaard EM, Hauer KE: A cross-sectional descriptive study of mentoring relationships formed by medical students. J Gen Intern Med 18:298-302, 2003

2. Aldrees T, Badri M, Islam T, Alqahtani K: Burnout among otolaryngology residents in Saudi Arabia: a multicenter study. J Surg Educ 72:844-848, 2015

3. Arora M, Diwan AD, Harris IA: Prevalence and factors of burnout among Australian orthopaedic trainees: a cross-sectional study. J Orthop Surg (Hong Kong) 22:374-377, 2014

4. Association of American Medical Colleges: Medical School Graduation Questionnaire. 2016 All Schools Summary Report. Washington DC: AAMC, 2016 (https://www.aamc. org/download/464412/data/2016gqallschoolssummaryreport. pdf) [Accessed January 4, 2018]

5. Balch CM, Shanafelt TD, Sloan JA, Satele DV, Freischlag JA: Distress and career satisfaction among 14 surgical specialties, comparing academic and private practice settings. Ann Surg 254:558-568, 2011

6. Becker JL, Milad MP, Klock SC: Burnout, depression, and career satisfaction: cross-sectional study of obstetrics and gynecology residents. Am J Obstet Gynecol 195:1444-1449, 2006

7. Bellini LM, Baime M, Shea JA: Variation of mood and empathy during internship. JAMA 287:3143-3146, 2002

8. Blanchard P, Truchot D, Albiges-Sauvin L, Dewas S, Pointreau Y, Rodrigues M, et al: Prevalence and causes of burnout amongst oncology residents: a comprehensive nationwide cross-sectional study. Eur J Cancer 46:2708-2715, 2010

9. Campbell DA Jr, Sonnad SS, Eckhauser FE, Campbell KK, Greenfield LJ: Burnout among American surgeons. Surgery 130:696-705, 2001

10. Castelo-Branco C, Figueras F, Eixarch E, Quereda F, Cancelo MJ, Gonzalez S, et al: Stress symptoms and burnout in obstetric and gynaecology residents. BJOG 114:94-98, 2007

11. Center C, Davis M, Detre T, Ford DE, Hansbrough W, Hendin $\mathrm{H}$, et al: Confronting depression and suicide in physicians: a consensus statement. JAMA 289:3161-3166, 2003

12. Chaput B, Bertheuil N, Jacques J, Smilevitch D, Bekara F, Soler P, et al: Professional burnout among plastic surgery residents: can it be prevented? Outcomes of a national survey. Ann Plast Surg 75:2-8, 2015

13. Dahlin ME, Runeson B: Burnout and psychiatric morbidity among medical students entering clinical training: a three year prospective questionnaire and interview-based study. BMC Med Educ 7:6, 2007

14. DeAngelis CD: Professors not professing. JAMA 292:10601061, 2004

15. DiMatteo MR, Sherbourne CD, Hays RD, Ordway L, Kravitz RL, McGlynn EA, et al: Physicians' characteristics influence patients' adherence to medical treatment: results from the Medical Outcomes Study. Health Psychol 12:93-102, 1993

16. Dorsey ER, Jarjoura D, Rutecki GW: Influence of control- 
lable lifestyle on recent trends in specialty choice by US medical students. JAMA 290:1173-1178, 2003

17. Dyrbye LN, Shanafelt TD: Physician burnout: a potential threat to successful health care reform. JAMA 305:2009_ 2010, 2011

18. Dyrbye LN, Shanafelt TD, Balch CM, Satele D, Sloan J, Freischlag J: Relationship between work-home conflicts and burnout among American surgeons: a comparison by sex. Arch Surg 146:211-217, 2011

19. Dyrbye LN, Thomas MR, Huntington JL, Lawson KL, Novotny PJ, Sloan JA, et al: Personal life events and medical student burnout: a multicenter study. Acad Med 81:374-384, 2006

20. Dyrbye LN, Thomas MR, Massie FS, Power DV, Eacker A, Harper W, et al: Burnout and suicidal ideation among U.S. medical students. Ann Intern Med 149:334-341, 2008

21. Dyrbye LN, Varkey P, Boone SL, Satele DV, Sloan JA, Shanafelt TD: Physician satisfaction and burnout at different career stages. Mayo Clin Proc 88:1358-1367, 2013

22. Dyrbye LN, West CP, Satele D, Boone S, Tan L, Sloan J, et al: Burnout among U.S. medical students, residents, and early career physicians relative to the general U.S. population. Acad Med 89:443-451, 2014

23. Eddleman CS, Aoun SG, Batjer HH: How to identify the edge of a cliff in the dark: burnout and neurosurgery. World Neurosurg 80:e111-e113, 2013

24. Elmore LC, Jeffe DB, Jin L, Awad MM, Turnbull IR: National survey of burnout among US general surgery residents. J Am Coll Surg 223:440-451, 2016

25. Epstein RM, Privitera MR: Doing something about physician burnout. Lancet 388:2216-2217, 2016

26. Fahrenkopf AM, Sectish TC, Barger LK, Sharek PJ, Lewin D, Chiang VW, et al: Rates of medication errors among depressed and burnt out residents: prospective cohort study. BMJ 336:488-491, 2008

27. Firth-Cozens J: Individual and organizational predictors of depression in general practitioners. Br J Gen Pract 48:16471651,1998

28. Firth-Cozens J, Greenhalgh J: Doctors' perceptions of the links between stress and lowered clinical care. Soc Sci Med 44:1017-1022, 1997

29. Fralick M, Flegel K: Physician burnout: who will protect us from ourselves? CMAJ 186:731, 2014

30. Freudenberger HJ: Staff burn-out. J Soc Issues 30:159-165, 1974

31. Golub JS, Weiss PS, Ramesh AK, Ossoff RH, Johns MM III: Burnout in residents of otolaryngology-head and neck surgery: a national inquiry into the health of residency training. Acad Med 82:596-601, 2007

32. Grayson MS, Newton DA, Thompson LF: Payback time: the associations of debt and income with medical student career choice. Med Educ 46:983-991, 2012

33. Healy NA, Glynn RW, Malone C, Cantillon P, Kerin MJ: Surgical mentors and role models: prevalence, importance and associated traits. J Surg Educ 69:633-637, 2012

34. Ishak WW, Lederer S, Mandili C, Nikravesh R, Seligman L, Vasa M, et al: Burnout during residency training: a literature review. J Grad Med Educ 1:236-242, 2009

35. Kassam A, Horton J, Shoimer I, Patten S: Predictors of wellbeing in resident physicians: a descriptive and psychometric study. J Grad Med Educ 7:70-74, 2015

36. Kimo Takayesu J, Ramoska EA, Clark TR, Hansoti B, Dougherty J, Freeman W, et al: Factors associated with burnout during emergency medicine residency. Acad Emerg Med 21:1031-1035, 2014

37. Klimo P Jr, DeCuypere M, Ragel BT, McCartney S, Couldwell WT, Boop FA: Career satisfaction and burnout among U.S. neurosurgeons: a feasibility and pilot study. World Neurosurg 80:e59-e68, 2013

38. Kuerer HM, Eberlein TJ, Pollock RE, Huschka M, Baile WF,
Morrow M, et al: Career satisfaction, practice patterns and burnout among surgical oncologists: report on the quality of life of members of the Society of Surgical Oncology. Ann Surg Oncol 14:3043-3053, 2007

39. Lockley SW, Cronin JW, Evans EE, Cade BE, Lee CJ, Landrigan $\mathrm{CP}$, et al: Effect of reducing interns' weekly work hours on sleep and attentional failures. N Engl J Med 351:1829_ 1837, 2004

40. Maslach CJS, Leiter MP: Maslach Burnout Inventory Manual, ed 3. Palo Alto: Consulting Psychologists Press, 1996

41. McAbee JH, Ragel BT, McCartney S, Jones GM, Michael LM II, DeCuypere M, et al: Factors associated with career satisfaction and burnout among US neurosurgeons: results of a nationwide survey. J Neurosurg 123:161-173, 2015

42. Merritt Hawkins: A Survey of America's Physicians: Practice Patterns and Perspectives. Dallas: Merritt Hawkins/The Physicians Foundation, 2012 (http://www. physiciansfoundation.org/healthcare-research/a-survey-ofamericas-physicians-practice-patterns-and-perspectives/) [Accessed January 4, 2018]

43. Nyssen AS, Hansez I, Baele P, Lamy M, De Keyser V: Occupational stress and burnout in anaesthesia. Br J Anaesth 90:333-337, 2003

44. Osborn EH: Factors influencing students' choices of primary care or other specialties. Acad Med 68:572-574, 1993

45. Palepu A, Friedman RH, Barnett RC, Carr PL, Ash AS, Szalacha L, et al: Junior faculty members' mentoring relationships and their professional development in U.S. medical schools. Acad Med 73:318-323, 1998

46. Pantaleoni JL, Augustine EM, Sourkes BM, Bachrach LK: Burnout in pediatric residents over a 2-year period: a longitudinal study. Acad Pediatr 14:167-172, 2014

47. Platz J, Hyman N: Mentorship. Clin Colon Rectal Surg 26:218-223, 2013

48. Quillin RC III, Pritts TA, Davis BR, Hanseman D, Collins JM, Athota KP, et al: Surgeons underestimate their influence on medical students entering surgery. J Surg Res 177:201206, 2012

49. Rafferty JP, Lemkau JP, Purdy RR, Rudisill JR: Validity of the Maslach Burnout Inventory for family practice physicians. J Clin Psychol 42:488-492, 1986

50. Ramanan RA, Taylor WC, Davis RB, Phillips RS: Mentoring matters. Mentoring and career preparation in internal medicine residency training. J Gen Intern Med 21:340-345, 2006

51. Ramondetta LM, Bodurka DC, Tortolero-Luna G, Gordinier M, Wolf JK, Gershenson DM, et al: Mentorship and productivity among gynecologic oncology fellows. J Cancer Educ 18:15-19, 2003

52. Rivera JA, Levine RB, Wright SM: Completing a scholarly project during residency training. Perspectives of residents who have been successful. J Gen Intern Med 20:366-369, 2005

53. Rohlfing J, Navarro R, Maniya OZ, Hughes BD, Rogalsky DK: Medical student debt and major life choices other than specialty. Med Educ Online 19:25603, 2014

54. Sciscione AC, Colmorgen GH, D'Alton ME: Factors affecting fellowship satisfaction, thesis completion, and career direction among maternal-fetal medicine fellows. Obstet Gynecol 91:1023-1026, 1998

55. Shanafelt TD, Balch CM, Bechamps GJ, Russell T, Dyrbye L, Satele D, et al: Burnout and career satisfaction among American surgeons. Ann Surg 250:463-471, 2009

56. Shanafelt TD, Boone S, Tan L, Dyrbye LN, Sotile W, Satele $\mathrm{D}$, et al: Burnout and satisfaction with work-life balance among US physicians relative to the general US population. Arch Intern Med 172:1377-1385, 2012

57. Shanafelt TD, Bradley KA, Wipf JE, Back AL: Burnout and 
self-reported patient care in an internal medicine residency program. Ann Intern Med 136:358-367, 2002

58. Shanafelt TD, Hasan O, Dyrbye LN, Sinsky C, Satele D, Sloan J, et al: Changes in burnout and satisfaction with work-life balance in physicians and the general US working population between 2011 and 2014. Mayo Clin Proc 90:1600-1613, 2015

59. Shanafelt TD, Mungo M, Schmitgen J, Storz KA, Reeves D, Hayes SN, et al: Longitudinal study evaluating the association between physician burnout and changes in professional work effort. Mayo Clin Proc 91:422-431, 2016

60. Shanafelt TD, West C, Zhao X, Novotny P, Kolars J, Habermann T, et al: Relationship between increased personal well-being and enhanced empathy among internal medicine residents. J Gen Intern Med 20:559-564, 2005

61. Spiotta AM, Fargen KM, Denham SL, Fulton ME, Kellogg $\mathrm{R}$, Young E, et al: Incorporation of a physical education and nutrition program into neurosurgery: a proof of concept pilot program. Neurosurgery 79:613-619, 2016

62. Steiner JF, Curtis P, Lanphear BP, Vu KO, Main DS: Assessing the role of influential mentors in the research development of primary care fellows. Acad Med 79:865-872, 2004

63. Teitelbaum HS, Ehrlich N, Travis L: Factors affecting specialty choice among osteopathic medical students. Acad Med 84:718-723, 2009

64. Tsen LC, Borus JF, Nadelson CC, Seely EW, Haas A, Fuhlbrigge AL: The development, implementation, and assessment of an innovative faculty mentoring leadership program. Acad Med 87:1757-1761, 2012

65. Tzischinsky O, Zohar D, Epstein R, Chillag N, Lavie P: Daily and yearly burnout symptoms in Israeli shift work residents. J Hum Ergol (Tokyo) 30:357-362, 2001

66. West CP, Huschka MM, Novotny PJ, Sloan JA, Kolars JC, Habermann TM, et al: Association of perceived medical errors with resident distress and empathy: a prospective longitudinal study. JAMA 296:1071-1078, 2006

67. West CP, Shanafelt TD, Kolars JC: Quality of life, burnout, educational debt, and medical knowledge among internal medicine residents. JAMA 306:952-960, 2011

68. Williams ES, Konrad TR, Scheckler WE, Pathman DE, Linzer M, McMurray JE, et al: Understanding physicians' intentions to withdraw from practice: the role of job satisfaction, job stress, mental and physical health. Health Care Manage Rev 26:7-19, 2001

69. Williams ES, Skinner AC: Outcomes of physician job satis- faction: a narrative review, implications, and directions for future research. Health Care Manage Rev 28:119-139, 2003

70. Wise MR, Shapiro H, Bodley J, Pittini R, McKay D, Willan A, et al: Factors affecting academic promotion in obstetrics and gynaecology in Canada. J Obstet Gynaecol Can 26:127-136, 2004

\section{Disclosures}

Dr. Schirmer has received support from the NIH/NINDS for non-study-related clinical or research effort, has ownership of NTI, and has received an honorarium from the AANS.

\section{Author Contributions}

Conception and design: Attenello, Buchanan, Wen, McCartney, Khalessi, Cohen-Gadol, Cheng, Mack, Schirmer, Swartz, Prall, Stroink, Giannotta, Klimo. Acquisition of data: Attenello, McCartney, Khalessi, Cohen-Gadol, Swartz, Prall, Stroink, Giannotta, Klimo. Analysis and interpretation of data: Attenello, Buchanan, Wen, Cohen-Gadol, Mack, Giannotta, Klimo. Drafting the article: Attenello, Buchanan, Wen, Donoho, Klimo. Critically revising the article: Attenello, Buchanan, Wen, Donoho, Khalessi, Mack, Swartz, Prall, Giannotta, Klimo. Reviewed submitted version of manuscript: all authors. Approved the final version of the manuscript on behalf of all authors: Attenello. Statistical analysis: Attenello, Buchanan, Wen, Cen, Mack, Klimo. Administrative/ technical/material support: Attenello, Cheng, Prall, Stroink, Giannotta, Klimo. Study supervision: Attenello, Prall, Stroink, Giannotta, Klimo.

\section{Supplemental Information}

\section{Online-Only Content}

Supplemental material is available with the online version of the article.

Appendix Table. https://thejns.org/doi/suppl/10.3171/2017.9. JNS17996.

\section{Correspondence}

Frank J. Attenello: Keck School of Medicine, University of Southern California, Los Angeles, CA. attenell@usc.edu. 